\title{
Mantissa Distributions
}

\section{By Alan G. Konheim}

Let $b$ be an integer, at least 2, and write each positive real number in the form (1)

$$
x=m b^{c},
$$

where $m$ (the mantissa) satisfies $1 / b \leqq m<1$ and $c$ (the characteristic) is an integer. We define the product of mantissas* $m_{1}$ and $m_{2}$ by

$$
m_{1} * m_{2}= \begin{cases}m_{1} m_{2} & \text { if } 1 / b \leqq m_{1} m_{2}<1, \\ b m_{1} m_{2} & \text { if } 1 / b^{2} \leqq m_{1} m_{2}<1 / b .\end{cases}
$$

Now suppose that $M_{1}$ and $M_{2}$ are independent, identically distributed random variables, each taking on values in the interval $[1 / b, 1)$ such that

$$
\operatorname{Pr}\left(M_{1} * M_{2} \leqq x\right)=\operatorname{Pr}\left(M_{1} \leqq x\right) .
$$

What are all of the possible choices for the distribution function of $M_{1}$ ? The answer is provided by the following

Theorem. $\operatorname{Pr}\left(M_{1} \leqq x\right)=F_{n}(x)$ or $F_{\infty}(x)(n=1,2, \cdots)$, where

$$
\begin{aligned}
F_{n}(x) & =\left\{\begin{array}{cl}
0 & \text { if }-\infty<x<b^{-1}, \\
1 / n & \text { if } b^{-1} \leqq x<b^{-1+(1 / n)}, \\
2 / n & \text { if } b^{-1+(1 / n)} \leqq x<b^{-1+(2 / n)}, \\
\vdots & \text { if } b^{-1} \leqq x<\infty, \\
1 & \text { if }-\infty<x<b^{-1}, \dagger \\
1+1 / n\left[n \frac{\log x}{\log b}+1\right] \text { if } b^{-1} \leqq x<1, \\
1 \quad \text { if } 1 \leqq x<\infty, \quad n=1,2, \cdots,
\end{array}\right.
\end{aligned}
$$

and

$$
F_{\infty}(x)=\left\{\begin{array}{l}
0 \quad \text { if }-\infty<x<b^{-1}, \\
1+\frac{\log x}{\log b}=\int_{1 / b}^{x} \frac{d u}{u \log b} \text { if } b^{-1} \leqq x<1, \\
1 \quad \text { if } 1 \leqq x<\infty .
\end{array}\right.
$$

Proof. We will write $M_{i}=b^{-\theta_{i}}(i=1,2)$, where $\Theta_{1}$ and $\Theta_{2}$ are independent, indentically distributed random variables, taking on values in $(0,1]$. Note that

$$
M_{1} * M_{2}=b^{-\left(\boldsymbol{\theta}_{1} \dot{+} \boldsymbol{\theta}_{2}\right)}
$$

Received June 22, 1964.

* If $m_{i}$ is the mantissa of $x_{i}$ then $m_{1} * m_{2}$ is the mantissa of $x_{1} x_{2}$.

$\dagger[$ ] denotes 'the integer part of.' 
where $\dot{+}$ denotes addition modulo one. Thus $(3)$ is equivalent to requiring that $\Theta_{1} \dot{+} \Theta_{2}$ and $\Theta_{1}$ have the same distribution. If we set

$$
\phi(n)=E\left\{e^{2 \pi i n \theta_{1}}\right\}=\int_{0}^{1} e^{2 \pi i n \theta_{1}} d F_{\Theta_{1}}\left(\theta_{1}\right),
$$

then (3) and the independence of $\Theta_{1}, \Theta_{2}$ imply

$$
\phi(n)=E\left\{e^{2 \pi i n\left(\boldsymbol{\theta}_{1}+\boldsymbol{\theta}_{2}\right)}\right\}=E\left\{e^{2 \pi i n\left(\boldsymbol{\theta}_{1}+\boldsymbol{\theta}_{2}\right)}\right\}=\phi^{2}(n)
$$

so that $\phi(n)=0$ or 1 . Certainly $\phi(0)=1$. There are two cases to be examined.

Case 1. $\phi(n)=0$ for all $n \neq 0$.

It follows from the uniqueness theorem for Fourier-Stieltjes series that $d F_{\boldsymbol{\theta}_{1}}\left(d \theta_{1}\right)=d \theta_{1}$ and hence $\operatorname{Pr}\left(M_{1} \leqq x\right)=F_{\infty}(x)$.

Case 2. $\phi(n)=1$ for some $n \neq 0$.

Let $m$ be the smallest positive integer such that $\phi(m)=1$. Then

$$
0=\int_{0}^{1}\left(1-e^{2 \pi i m \theta_{1}}\right) d F_{\Theta_{1}}\left(\theta_{1}\right)=\int_{0}^{1}\left(1-\cos 2 \pi m \theta_{1}\right) d F_{\Theta_{1}}\left(\theta_{1}\right) .
$$

It follows that $F_{\boldsymbol{\theta}_{1}}$ is a 'step function' with points of discontinuity at $\theta_{k}=k / m$ $(k=1,2, \cdots, m)$ and, hence, $\phi(n+m)=\phi(n)(n=0, \pm 1, \pm 2, \cdots)$. We assert that $\phi(n)=1$ if and only if $n=k m$ for some integer $k$; for if $\phi(n)=1$ with $k m<n<(k+1) m$ then $\phi(n-k m)=\phi(n)=1$ while $0<n-k m<m$ contradicting the minimality of $m$. The uniqueness theorem for Fourier-Stieltjes series now shows that $\operatorname{Pr}\left(M_{1} \leqq x\right)=F_{m}(x)$.

I should like to acknowledge with thanks several suggestions made by $\mathrm{Mr}$. Benjamin Weiss.

Thomas J. Watson Research Center

International Business Machines Corporation

Yorktown Heights, New York

\section{New Primes of the Form $n^{4}+1$}

\section{By A. Gloden}

This note presents some results of a continuation of the author's systematic factorization of integers of the form $n^{4}+1$ [1].

An electronic computer at l'Institut Blaise Pascal in Paris has been used to find solutions of the congruence $x^{4}+1 \equiv 0(\bmod p)$ for all primes of the form $8 k+1$ in the interval $10^{6}<p<4 \cdot 10^{6}$, thereby extending the previous range of such tables listed in [1].

With the aid of these tables, the complete factorization of $n^{4}+1$ has now been effected for all even values of $n$ less than 2040 and for all odd values less than 2397 .

Thus, the primality of $\frac{1}{2}\left(n^{4}+1\right)$ has been established for the following 116 values of $n$ :

Received February 25, 1963. Revised August 2, 1963. 